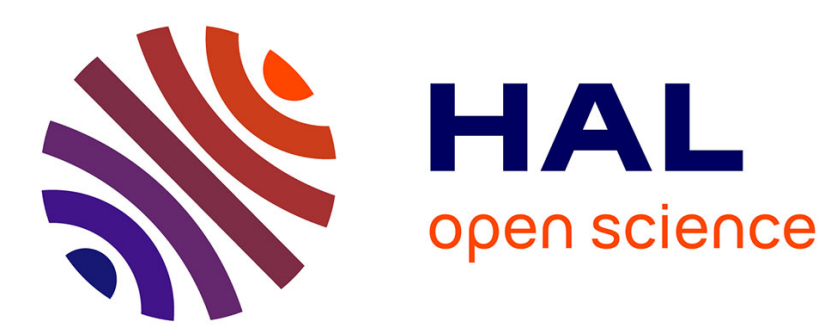

\title{
Analyse automatique de conversations textuelles synchrones d'apprenants pour la détermination de comportements sociaux
}

Sébastien George

\section{- To cite this version:}

Sébastien George. Analyse automatique de conversations textuelles synchrones d'apprenants pour la détermination de comportements sociaux. STICEF (Sciences et Technologies de l'Information et de la Communication pour l'Éducation et la Formation), 2004, Vol. 10, p. 165-193. hal-00298117

\section{HAL Id: hal-00298117 \\ https://hal.science/hal-00298117}

Submitted on 16 Jul 2008

HAL is a multi-disciplinary open access archive for the deposit and dissemination of scientific research documents, whether they are published or not. The documents may come from teaching and research institutions in France or abroad, or from public or private research centers.
L'archive ouverte pluridisciplinaire HAL, est destinée au dépôt et à la diffusion de documents scientifiques de niveau recherche, publiés ou non, émanant des établissements d'enseignement et de recherche français ou étrangers, des laboratoires publics ou privés. 

pour la détermination de comportements sociaux,

Article envoyé pour publication dans la Revue STICEF, numéro spécial « technologies et formation à distance », INRP, Vol. 10, 2003, (p. 165-193)

\title{
Analyse automatique de conversations textuelles synchrones d'apprenants pour la détermination de comportements sociaux
}

\author{
Sébastien George \\ Laboratoire ICTT (Interaction Collaborative, Téléformation, Téléactivités) \\ 21, avenue Jean Capelle \\ 69621 Villeurbanne Cedex - France \\ Sebastien.George@insa-lyon.fr
}

\begin{abstract}
RÉSUMÉ. Dans cet article, nous nous intéressons à l'apprentissage collectif dans un contexte de distance et à l'utilisation des technologies informatiques pour le soutenir. Une des limites de la distance réside dans la difficulté d'avoir un retour d'information sur les actions et comportements des utilisateurs. En formation à distance, cette difficulté est un obstacle pour le suivi pédagogique des tuteurs et pour la réalisation d'activités collectives entre apprenants. Nous pensons que l'informatique peut contribuer à résoudre ce problème en recueillant des informations sur le comportement des utilisateurs. En particulier, nous proposons ici une approche pour la détermination automatique de comportements sociaux, comme des comportements d'animateurs ou d'indépendant, lors de conversations textuelles synchrones. Cette approche est fondée sur la spécification et la conception d'un outil de conversation semi-structuré par des actes de langage.
\end{abstract}

ABSTRACT. In this paper, we deal with collective learning in a distance context and with information technology to support it. A limit of distance situation leads in the difficulty to have a feedback on users' actions and behaviors. In distance education, this difficulty is an obstacle to the monitoring of teacher and to the collective work of learners. We think that computer system can contribute to solve this problem by giving feedback on users' behavior. We propose an approach to automatic analysis of learners' social behavior, like moderator or independent, during computer-mediated synchronous textual conversations. This approach is based on the specification and design of a conversation tool semi-structured by communicative acts.

MOTS-CLÉS : formation en ligne, apprentissage collectif, analyse automatique de conversations médiatisées, profils de comportementaux sociaux.

KEYWORDS: e-learning, collective learning, automatic analysis of computer-mediated conversations, social behavior profiles 


\section{Introduction}

Notre travail se situe dans le domaine des Environnements Informatiques pour l'Apprentissage Humain (EIAH) et concerne plus spécifiquement les EIAD (Environnements Interactifs pour l'Apprentissage à Distance). Nous définissons les EIAD comme étant des environnements cherchant à créer des conditions de construction de connaissances chez une personne à partir d'interactions avec un système informatique distribué et d'interactions médiatisées avec d'autres acteurs (enseignants, autres apprenants) utilisant ce système.

Le contexte général de notre travail concerne l'apprentissage collectif dans un contexte de distance et l'utilisation des technologies informatiques pour le soutenir. Nous définissons l'apprentissage collectif comme étant un mode de partage et de construction de connaissances se réalisant lors d'interactions entre apprenants. Nous nous fondons pour cela sur des approches en psychologie du développement comme l'approche socio-culturelle [Vygotski34] et l'approche socio-constructiviste [DoiseMugny81]. Ces approches sont appuyées par des approches pédagogiques [Dewey22] [Freinet77]. Dans toutes ces approches, l'enseignant est un acteur important qui est considéré comme un initiateur d'activités collectives favorisant l'apprentissage entre pairs, comme un facilitateur et un régulateur.

Nous nous intéressons dans cet article à l'Apprentissage Collectif Assisté par Ordinateur (ACAO) plus connu sous sa dénomination anglaise Computer-Supported Collaborative Learning (CSCL). Nous posons comme hypothèse fondatrice que les situations d'apprentissage à distance utilisant les réseaux informatiques peuvent, sous certaines conditions, être un levier puissant pour favoriser l'apprentissage collectif. Nous avons adopté une démarche de recherche consistant à déterminer en premier lieu l'activité pédagogique collective à mettre en place. En effet, il ne faut pas seulement compter sur les nouvelles technologies de la communication pour créer des situations d'apprentissage collectif. Les conditions qui nous paraissent primordiales pour favoriser l'apprentissage collectif sont la création d'interdépendance entre apprenants, la recherche d'un apprentissage par l'action et la mise en place d'activités de moyenne durée - plusieurs semaines à quelques mois - devant aboutir à une production collective. Dans notre cas, nous avons choisi la pédagogie de projet comme fondement pour favoriser l'apprentissage collectif. Une partie de notre travail a alors consisté à expliciter et à modéliser une activité de projet pour un contexte d'apprentissage à distance. Notre modèle s'appuie sur une organisation humaine en équipe, cette dernière étant composée d'apprenants se trouvant à distance et engagés dans un même projet. Un tuteur, distant lui aussi, assure le suivi du projet et assiste les apprenants. Le résultat de ce travail de modélisation est présenté dans [GeorgeLeroux01].

Dès lors, nous avons déterminé les spécifications d'un environnement informatique ayant pour objectif de soutenir les acteurs d'une pédagogie de projet dans un contexte de distance. Cet environnement est un support dans le sens où il est 
conçu pour la mise en place, pour la réalisation et pour le suivi d'activités de projets entre apprenants. L'intérêt d'avoir conçu cet environnement est double. D'une part, l'environnement est dédié à la pédagogie de projet, approprié au contexte éducatif et utilisable par un large public d'apprenants. D'autre part, son architecture et ses outils permettent l'analyse automatique des activités des acteurs afin de les assister. L'environnement, nommé SPLACH (Support d'une pédagogie de Projet pour l'Apprentissage Collectif Humain), est décrit dans [George01] ou de manière plus synthétique dans [George02] et [GeorgeLeroux02].

Dans le cadre de l'analyse automatique des activités collectives des apprenants, nous proposons une approche particulière pour analyser les conversations synchrones entre apprenants, ces interactions étant révélatrices de comportements sociaux - animateur, indépendant, etc. - qu'il nous paraît important de pouvoir repérer lors d'une pédagogie de projet. Cette analyse automatique de comportements sociaux est l'objet de cet l'article. Pour notre travail, nous nous intéressons aux conversations médiatisées synchrones de forme textuelle, plus communément appelé chat.

Nous précisons dans la partie 2 de l'article les objectifs visés dans la détermination de profils de comportements sociaux. La partie 3 présente les travaux de Pléty sur lesquels nous nous appuyons pour étudier les comportements sociaux d'apprenants. La partie 4 décrit nos fondements théoriques et pratiques pour l'analyse automatique de conversations. Nous présentons alors dans la partie 5 notre proposition pour la détermination automatique de profils de comportements sociaux lors de conversations textuelles synchrones. Cette proposition s'est concrétisée par la conception d'un outil de conversation synchrone particulier dont l'évaluation est décrite dans la partie 6.

\section{Objectifs recherchés dans la détermination de profils de comportements sociaux}

Lorsque des apprenants travaillent en groupe dans une classe, une organisation se met en place spontanément. Pléty précise qu' « au sein de cette organisation chacun des partenaires sait trouver, suivant ses capacités et ses connaissances personnelles, sa propre place »Les apprenants se positionnent les uns par rapport aux autres en fonction de comportements sociaux révélés par les attitudes de chacun. L'observation de ces comportements sociaux des apprenants dans une classe est une chose que tout professeur fait de façon habituelle. Dans le cadre d'une pédagogie de projet par exemple, ces observations fournissent à l'enseignant encadrant des indicateurs pour comprendre, réagir et intervenir auprès du groupe. De même, pour les apprenants, la perception des comportements des individus du groupe permet de mieux réguler le travail collectif.

Dans une situation de distance, des caractéristiques de comportement social similaires existent dans les groupes distribués [Rheingold93]. Cependant, la distance et la médiatisation des échanges ne rendent pas aussi immédiat la perception de ces 
comportements sociaux. Pour pallier cette limitation, nous pensons qu'il est utile d'avoir un système informatique capable d'analyser automatiquement ces comportements. En particulier, dans un contexte de formation en ligne, il est intéressant de chercher à déterminer automatiquement des profils de comportements sociaux à l'intérieur des groupes d'apprenants car ils peuvent être utiles aussi bien pour le tuteur que pour les apprenants eux-mêmes.

Pour le tuteur responsable de l'encadrement des apprenants lors d'une formation à distance, des informations sur des profils de comportements sociaux lui permettraient de mieux comprendre les relations sociales existantes. Dans le cas d'activités pédagogiques collectives, le tuteur pourrait alors mieux suivre l'activité qui se déroule et donc mieux encadrer les groupes d'apprenants. D'une manière générale, nous pensons que des indications sur les comportements sociaux des apprenants peuvent contribuer à aider le tuteur dans son suivi pédagogique, suivi qui constitue un point crucial pour la bonne réussite d'une formation à distance. En particulier, le suivi d'un tuteur aide à limiter les abandons liés au sentiment d'isolement des apprenants [Linard01]. Pouvoir repérer rapidement un apprenant isolé est un enjeu important. Notre proposition est donc de soutenir informatiquement le suivi du tuteur. Le tuteur ne peut pas être présent à chaque connexion des apprenants. Un système informatique l'assistant dans sa tâche de suivi sera, dans ce contexte, d'une aide précieuse. L'objectif est de concevoir des systèmes présentant des vues synthétiques des informations pertinentes pour le tuteur [Labat02]. Toujours du côté des enseignants, nous voyons d'autres perspectives possibles à l'utilisation des profils de comportement. Par exemple, ces profils peuvent constituer une aide à la formation de groupes d'apprenants. Un enseignant aurait la possibilité d'utiliser les profils en cherchant à « équilibrer » un groupe ou, au contraire, en cherchant à créer des conflits intéressants d'un point de vue pédagogique [Dillenbourganda196]).

En ce qui concerne les apprenants, leur fournir des informations sur leurs profils de comportement social permettrait de leur donner une vue sur leurs actions et leur donner ainsi un autre regard sur leur processus de travail. Cet « effet miroir » est l'une des aides possibles d'un environnement support d'apprentissage collectif [Jermannandal01]. Nous pouvons même aller au-delà et utiliser les profils analysés pour adapter les environnements aux apprenants. Par exemple, un apprenant ayant un comportement d'animateur se verrait attribuer des outils particuliers d'animation. Par ailleurs, une analyse automatique aboutissant à la détermination de profils comportementaux contribuerait à fournir des indicateurs au système informatique pour apporter de l'assistance adaptée et des conseils personnalisés aux apprenants.

Nous venons de citer les multiples intérêts d'une analyse automatique des profils de comportements sociaux dans un contexte de formation à distance. La question est de savoir comment parvenir à cette analyse automatique. Nous présentons l'état de nos travaux sur une approche pour y parvenir. Pour notre travail, nous nous attachons uniquement à l'observation et à l'analyse des comportements sociaux pendant les conversations textuelles synchrones. 


\section{Fondements pour l'étude de comportements sociaux d'apprenants}

Robert Pléty, chercheur du laboratoire d'éthologie des communications de l'université Lumière-Lyon 2, a beaucoup étudié le comportement d'élèves travaillant en groupe dans une classe ; il a notamment analysé des interactions entre élèves ${ }^{1}$ travaillant en groupe de quatre à la résolution de problèmes d'algèbre [Pléty96]. Pour ce faire, il est parti d'une micro-analyse des échanges verbaux et gestuels dans le groupe pour aboutir à la détermination de profils de comportement chez les élèves. De cette analyse, Pléty a défini des profils de comportement qui caractérisent les rôles que les partenaires jouent dans le groupe. Ses analyses lui ont permis d'identifier des profils types de comportement. Afin de déterminer ces comportements quatre sortes d'observations sont effectuées pour chaque élève : le volume d'intervention, le type d'intervention, les gestes de type communicatif (regard et mouvement), les réactions des autres (ce que les interventions entraînent).

À partir de ces observations, Pléty fait ressortir quatre profils types de comportement: l'animateur, le vérificateur, le quêteur et l'indépendant. Les caractéristiques de ces quatre profils sont résumées dans le Tableau 1.

Tableau 1. Les profils comportementaux d'élèves travaillant en groupe (synthèse personnelle d'après les travaux de Pléty)

\begin{tabular}{|l|l|l|l|l|}
\hline Profil & \multicolumn{1}{|c|}{$\begin{array}{c}\text { Volume } \\
\text { d'intervention }\end{array}$} & \multicolumn{1}{c|}{$\begin{array}{c}\text { Type } \\
\text { d'intervention }\end{array}$} & $\begin{array}{c}\text { Gestes } \\
\text { communicatifs }\end{array}$ & \multicolumn{1}{c|}{$\begin{array}{c}\text { Réactions } \\
\text { entraînées }\end{array}$} \\
\hline Animateur & Important & $\begin{array}{l}\text { Interrogation ou } \\
\text { proposition }\end{array}$ & $\begin{array}{l}\text { Importants (regards } \\
\text { et mouvements) }\end{array}$ & $\begin{array}{l}\text { Suivi de réactions } \\
\text { positives }\end{array}$ \\
\hline Vérificateur & Assez important & $\begin{array}{l}\text { Réaction, réponse et } \\
\text { évaluation }\end{array}$ & $\begin{array}{l}\text { Régulateurs (regards } \\
\text { orientés) }\end{array}$ & $\begin{array}{l}\text { Peu suivi de } \\
\text { réactions }\end{array}$ \\
\hline Quêteur & Peu important & $\begin{array}{l}\text { Très dubitatif } \\
\text { (questions) }\end{array}$ & $\begin{array}{l}\text { Regards longs de } \\
\text { quête }\end{array}$ & $\begin{array}{l}\text { Ses questions sont } \\
\text { bien acceptées }\end{array}$ \\
\hline Indépendant & Faible & $\begin{array}{l}\text { Pas ou peu de propo- } \\
\text { sition ni d'évaluation }\end{array}$ & Peu de regards & $\begin{array}{l}\text { Ses interventions } \\
\text { restent en suspens }\end{array}$ \\
\hline
\end{tabular}

D'après cette étude, ces quatre profils se retrouvent dans presque tous les groupes analysés (16 au total). Ainsi, un animateur est présent dans tous les groupes analysés. Un vérificateur est presque toujours présent (dans 12 des 16 groupes) et ce rôle n'est qu'une seule fois conjoint au rôle d'animateur. Un quêteur est présent dans tous les groupes et il arrive même d'en retrouver plusieurs dans un même groupe. Un indépendant se retrouve un peu moins fréquemment (dans 9 des 16 groupes).

Nous nous sommes alors posés la question de savoir si, à distance et avec les réseaux, il était possible de retrouver ces mêmes caractéristiques de groupes d'apprenants. Pléty apporte un élément de réponse, parlant de groupes d'apprenants

1. Les élèves de son étude étaient des collégiens en classe de $4^{\text {ème }}$. 
distribués sur réseau, en soulignant que « curieusement, on y rencontre les mêmes aspects d'appartenance, de cohésion et de leadership que dans les groupes ordinaires »[Pléty98]. La détermination de profils de comportement pour des apprenants travaillant en groupe à distance semble donc avoir un sens. La question est alors de savoir comment transposer les travaux de Pléty dans un contexte de conversation médiatisée. Une des manières d'y arriver est d'analyser automatiquement les conversations entre apprenants.

\section{Fondements pour l'analyse automatique de conversations}

Nous avons vu précédemment qu'il était possible de déterminer des profils comportementaux en analysant «manuellement» les interactions et les conversations lors de réunions de groupe. Notre objectif est d'automatiser la détermination de ces profils comportementaux en concevant un système d'analyse des conversations. Pour réaliser ces analyses nous avons besoin de comprendre plus en détail ce qu'est une conversation. Nous présentons dans cette partie les travaux sur lesquels nous nous fondons pour atteindre notre objectif consistant à déterminer automatiquement des profils de comportements sociaux lors de conversations textuelles synchrones.

\subsection{La théorie des actes du langage}

L'étude de la fonction du langage a fait l'objet de nombreuses recherches. Pendant longtemps, le langage a été perçu comme étant un moyen de rendre compte et de décrire le monde. Austin [Austin62] présente une autre vision du langage en considérant les énoncés d'une conversation comme étant non plus seulement descriptifs mais comme des actes en soi. Les énoncés sont eux-mêmes des actions et possèdent une force illocutoire, c'est-à-dire une certaine valeur (question, proposition, etc.). Ainsi, Austin pose comme principe de la théorie des actes de langage qu'en énonçant un message un locuteur effectue simultanément trois actes :

- un acte locutoire : c'est l'action même de dire quelque chose ;

- un acte illocutoire : c'est la valeur conventionnelle du message ;

- un acte perlocutoire : c'est l'ensemble des conséquences qui découlent de l'acte de parole (les effets).

Pour illustrer ce principe, nous pouvons prendre l'exemple d'une personne qui dit : «Peux-tu fermer la porte ? ». La personne prononce une phrase (aspect locutoire) pour demander quelque chose (aspect illocutoire) dans le but d'avoir un effet sur la personne à qui ce message est destiné (aspect perlocutoire). L'effet attendu (la fermeture de la porte) n'est pas forcément celui qui va être réalisé (par exemple une réponse de l'interlocuteur comme « Vas-y toi-même !»).

De nombreux travaux se sont fondés sur la théorie des actes de langage d'Austin. Parmi ceux-ci, l'approche interactionnelle des actes de langages nous semble particulièrement intéressante pour l'analyse des conversations personne-personnes médiatisées. 


\subsection{Approche interactionnelle des actes de langage}

L'approche interactionnelle de la conversation a fortement été influencée par le courant de pensée dit de "l'école genevoise ». Les chercheurs de ce courant ont défini un modèle structurel et fonctionnel du dialogue ${ }^{2}$. Les intérêts de ce modèle proviennent du fait, d'une part, qu'il se veut indépendant de la tâche et, d'autre part, qu'il permet d'identifier les différents types de constituants d'une conversation : les échanges, les interventions et les actes de langage [Rouletandal85]. Selon ces derniers auteurs, un dialogue consiste en une suite d'échanges constitués eux-mêmes par des interventions, qui sont à leurs tours composées d'actes de langage. Ce modèle structurel et fonctionnel genevois " est basé sur l'hypothèse que la conversation est organisée à partir d'un ensemble hiérarchisé d'unités de rang et de relations ou fonctions entre ces unités » [Moeschler94].

Comme nous l'avons dit, une intervention peut se composer d'un ou plusieurs actes de langage. Cependant, il est communément admis qu'il y a au moins un acte qui est l'acte principal ou acte directeur [Rouletandal85] qui détermine la force illocutoire de l'intervention. Selon Bilange, « les interventions peuvent avoir trois fonctions illocutoires génériques : l'initiative, la réaction et l'évaluation » [Bilange91]. Cette force illocutoire de l'intervention est entièrement déterminée par l'acte de langage directeur de l'intervention. De ce fait, les actes de langage peuvent être classifiés en actes initiatifs, en actes réactifs et en actes évaluatifs. Nous préférons cette classification très générale à celle plus fine de Searle [Searle72] par exemple. En effet, nous trouvons cette classification plus pertinente au regard de notre objectif concernant la détermination des profils comportementaux. Nous retrouvons dans ces trois catégories - que sont les initiatifs, les réactifs et les évaluatifs - les indicateurs potentiels de comportements identifiés par Pléty. Nous y reviendrons dans la partie 4.2 lorsque nous définissons précisément les actes de langage de notre outil de conversation.

D’une manière générale, les chercheurs du courant de l'école genevoise critiquent les théories fondées sur les seuls actes de langage conçus comme des actes isolés. Ils mettent en avant l'idée d'échanges entre personnes et de séquences structurées d'échanges. Pour Roulet [Rouletanda185], une intervention doit remplir un certain nombre de conditions pour constituer une réaction adéquate et autoriser la poursuite de l'échange. Cela ne veut pas dire pour autant qu'il existe des règles strictes qui s'appliquent au déroulement d'une conversation. Néanmoins, nous ne pouvons ignorer l'existence de certaines contraintes d'enchaînements [Moeschler94]. Il existe par exemple des contraintes thématiques qui sont des contraintes liées aux contenus des interventions. La contrainte qui nous paraît

2. Ce modèle du dialogue, donc a priori entre deux personnes, est aussi pertinent pour servir de modèle à toute conversation impliquant plus de deux locuteurs. D'ailleurs ce modèle est aussi parfois appelé «modèle conversationnel ». 
intéressante est celle que Roulet appelle la « condition illocutoire » qui, selon lui, « impose au constituant réactif une fonction illocutoire correspondant à celle du constituant initiatif »[Rouletandal85]. L'exemple le plus simple peut se retrouver dans les deux principaux types d'échanges identifiés par Kerbrat-Orecchioni [Kerbrat-Orecchioni92] : les échanges minimaux qui sont composés d'une intervention initiative suivie d'une intervention réactive, et les échanges complets qui comportent en plus une intervention évaluative. Cette vue est assez macroscopique et nous traitons plus finement des contraintes d'enchaînement conversationnel dans la partie suivante.

\subsection{Enchaînement conversationnel}

Le principal objectif des recherches concernant les enchaînements conversationnels est de trouver des structures récurrentes dans les conversations entre personnes. La conversation est vue comme une co-construction dans laquelle les interlocuteurs interagissent de manière cohérente. D'une manière générale, nous retenons que « chaque interaction répond à une interaction précédente et en même temps impose des contraintes discursives sur l'interaction suivante » [ColineauMoulin99]. Dans une perspective d'assistance à la structuration de conversation, nous pensons qu'il est utile de prendre en compte ces contraintes discursives qui permettent de restreindre pour chaque énoncé l'ensemble des énoncés pouvant intervenir à la suite.

Les travaux de Clark et Schaefer [ClarkSchaefer89] nous donne un éclairage notoire sur les enchaînements conversationnels. Ces auteurs ont beaucoup étudié ce qu'ils nomment les « contributions » dans une conversation. Ils ont défini un modèle des contributions dans lequel celles-ci sont créées par les participants agissant collectivement : " contributions are created by participants acting collectively » [ClarkSchaefer89]. Les contributions sont certes individuelles, mais dépendantes des contributions des autres participants. Toujours selon ces chercheurs, la plupart des tours de conversation sont organisés en paires adjacentes ${ }^{3}$. Une paire adjacente se compose de deux expressions ordonnées produites par deux locuteurs. L'exemple le plus simple de paire adjacente est la paire « question-réponse ». La forme et le contenu de la deuxième partie de la paire (la réponse) dépendent du type de la première partie de la paire (la question). Une première partie d'une paire étant donnée, une seconde est appropriée et attendue à la prochaine expression : «Given a first pair part, a second pair part is conditionally relevant, that is, relevant and expectable, as the next utterance » [ClarkSchaefer89]. Clark et Shaefer proposent ainsi des types de paires adjacentes possibles.

3. Adjacency Pairs en anglo-saxon. 
L'organisation en paires adjacentes rend compte de la structuration de la conversation. Un premier acte communicatif entraîne une réaction appropriée de l'interlocuteur. Cette organisation en paires adjacentes a l'avantage d'être très structurante. Nous pouvons aussi y voir une limite. En effet, dans les travaux de Clark et Schaefer, à une première partie de paire ne correspond qu'une seule deuxième partie. Cette vision nous semble quelque peu restrictive. Dans la partie 4.3, nous proposons des contraintes d'enchaînements qui s'inspirent de ces paires adjacentes mais présentent plus de souplesse en donnant différents choix pour la poursuite de la conversation.

\subsection{Conversations médiatisées semi-structurées par des actes de langage}

Nous avons vu qu'une conversation pouvait être considérée comme une succession d'échanges composés d'interventions de locuteurs et constituées ellesmêmes d'actes de langage dont l'un est l'acte directeur. Si l'on cherche à analyser automatiquement une conversation, il est alors important de repérer ces actes de langage directeurs. Deux solutions s'offrent alors à nous. La première consiste à analyser le contenu des interventions pour tenter de déterminer ces actes. C'est le domaine du traitement automatique du langage naturel (TALN). Dans son livre, Luzzati [Luzzati95] relève les difficultés de ces analyses automatiques et notamment celle de la compréhension du sens par le système. Malgré les progrès effectués dans ce domaine, les résultats ne sont pas toujours très fiables. Les systèmes les plus performants sont ceux qui analysent des conversations faites dans un contexte très précis et très limité. De ce fait, beaucoup de travaux concernés par cette analyse automatique du langage se trouvent dans le domaine du dialogue personne-machine finalisé.

La deuxième solution pour déterminer l'acte de langage directeur dans une intervention est de le demander à l'utilisateur. C'est un principe de base qui est notamment utilisé dans le domaine de l'EIAH : si le système ne sait reconnaître l'information nécessaire pour son analyse alors il faut la demander à l'utilisateur : «avoid guessing, get the student to tell you what you need to know $»^{4}$ [Self88]. Une solution consiste alors à utiliser une interface semi-structurée pour demander un acte de langage à l'utilisateur avant que celui-ci ne saisisse le contenu de son message de manière libre (d'où le terme de semi-structuration). Le premier système à avoir utilisé ce principe pour obtenir des informations à propos des actes de langage est un système pour le travail coopératif assisté par ordinateur (TCAO), nommé The Coordinator [Winograd87]. The Coordinator est un système pour la gestion des actions dans le temps et comporte un système de messagerie composé de menus permettant d'identifier les actions linguistiques. Les menus sont adaptés aux

4. « N'essayez pas de deviner, demander à l'apprenant de vous dire ce que vous avez besoin de savoir » (traduction personnelle) 
messages et à l'état de la conversation. L'objectif principal est de faciliter l'identification de rupture dans la coordination des personnes. Selon les auteurs, The Coordinator a aussi une dimension éducative : "The Coordinator also has an educational dimension »[Floresandal88]. En effet en utilisant le système, les personnes acquièrent de nouvelles compétences en matière d'action sociale. Le simple fait de caractériser un message comme une «offre » ou une «demande » amène l'utilisateur à se demander « qu'est-ce que je veux faire là ? ». De nombreux environnement d'ACAO ont alors repris le principe de semi-structuration des communications, entres autres ICLS [McManusAiken95], C-CHENE [BakerLund97], TecfaMOO [JermannSchneider97], BetterBlether [Robertsonandal98] et COMET [Solleranda199].

Même si cette technique comporte des inconvénients, comme le manque de flexibilité ou l'ajout de contraintes et de discipline pour l'utilisateur [Suchman94], elle peut être utile dans un contexte d'apprentissage pour les raisons suivantes :

- le fait de typer un message amène l'utilisateur à se demander ce qu'il veut dire et a donc une valeur éducative [Winograd87] [Floresandal88] ;

- une interface semi-structurée encourage les utilisateurs à se centrer davantage sur la tâche [BakerLund97] [JermannSchneider97] ;

- l'utilisation d'ouvreurs permet de concevoir des systèmes d'analyse automatique de discussions [McManusAiken95].

Il nous semble donc opportun d'utiliser une structuration au moyen d'actes de langage pour, d'une part, concevoir un outil de conversation dans un contexte éducatif et, d'autre part, analyser automatiquement des profils de comportements sociaux. Nous sommes conscients des critiques qui existent à ce sujet et nous tentons d'apporter des solutions notamment par rapport aux points suivants :

- la structuration de l'environnement doit par elle-même encourager à utiliser l'acte de langage correspondant à l'intervention [Robertsonanda198] ;

- un grand nombre d'actes rend l'interface difficilement utilisable [Sollerandal99];

- l'outil doit permettre de rendre le fil des conversations explicite [Robertsonandal98].

\section{Proposition pour la détermination de profils de comportements sociaux}

Nous présentons dans cette partie notre proposition pour déterminer de manière automatique, lors de conversations textuelles synchrones, des profils de comportement sociaux.

\subsection{Réflexion pour le choix des actes de langage pour l'outil de conversation}

Nous poursuivons un double objectif en proposant une interface semi-structurée par des actes de langage pour l'outil de conversation. D'une part, nous souhaitons 
analyser automatiquement certains aspects des conversations afin de faire ressortir des profils comportementaux des utilisateurs. En nous fondant sur les travaux de Pléty et en les transposant dans notre contexte de conversation médiatisée, les indicateurs permettant de déterminer ces profils peuvent être considérés comme étant de deux natures : d'une nature quantitative en ce qui concerne le volume des interventions et d'une nature qualitative pour ce qui est du type d'intervention. Les actes de langage nous intéressent pour ce deuxième point. Les actes de notre système sont choisis relativement aux indicateurs de type d'intervention relevés par Pléty (colonne «type d'intervention »du Tableau 1) à savoir : interrogation, proposition, réaction, réponse, évaluation, question. Ces types constituent les premiers éléments de définition des actes pour notre outil.

D'autre part, le second objectif est de se servir de la structuration qu'implique l'utilisation des actes de langage pour faciliter la conversation des apprenants. Ainsi, nous pensons que l'outil proposé, plutôt que d'être un obstacle, doit permettre d'améliorer les conversations médiatisées.

Afin de limiter les choix possibles d'actes de langage et donc la complexité, nous choisissons de ne pas utiliser une classification théorique des actes de langage mais plutôt une classification pragmatique en rapport avec nos besoins. Les taxinomies sont utiles pour des chercheurs mais sont peu adaptées à des utilisateurs qui veulent communiquer (les actes proposés ne sont pas assez concrets). Il est certain que le fait de définir un ensemble d'actes de langage comporte une part d'arbitraire. Des processus itératifs de tests auprès des utilisateurs (durant une première expérimentation) nous ont permis d'affiner l'ensemble d'actes de langages déterminés de manière pragmatique.

Nous choisissons de présenter les actes de langage sous forme de verbes. En effet, un verbe est plus proche de l'action de l'utilisateur qui veut exprimer quelque chose [Scapin86]. Notre choix s'oriente vers un ensemble de verbes qui expriment des forces illocutoires. De plus, les verbes choisis doivent relever d'un vocabulaire usuel et ne pas faire appel à des définitions complexes ou ambiguës.

D'un point de vue ergonomique, la présentation des actes dans un menu déroulant lorsque l'utilisateur sélectionne un message de la conversation semble la plus « utilisable » et la moins déroutante pour les utilisateurs. Un autre avantage de ce menu est qu'il peut être créé de façon contextuelle : selon le message auquel l'utilisateur réagit, nous pouvons lui présenter la liste des actes pour enchaîner.

\subsection{Les actes de langage proposés pour l'outil de conversation}

Les actes que nous proposons d'intégrer dans l'outil de conversation semistructuré se répartissent en cinq catégories. Trois de ces catégories se trouvent par exemple dans les travaux de [Roulet86] à savoir : les actes initiatifs, les actes réactifs et les actes évaluatifs (cf. partie 3.2). Nous avons ajouté deux autres catégories à celles-ci. La première est la catégorie « salutation » [KendonFerber73] [Laver81] qui permet de commencer et de clore une conversation. La deuxième catégorie ajoutée 
est celle des actes auto-réactifs. Nous n'avons pas trouvé de travaux y faisant référence. Nous pensons néanmoins qu'il est nécessaire de pouvoir réagir à ses propres interventions. Il est vrai que cette catégorie peut se confondre avec la catégorie des actes réactifs. Cependant, nous préférons distinguer clairement les interventions de réaction d'un locuteur à l'une de ses propres interventions, de celles réagissant à des interventions émises par d'autres. Le Tableau 2 précise les actes que nous avons identifiés pour chacune des catégories retenues.

Tableau 2. Les actes de langage proposés pour notre outil de conversation

\begin{tabular}{|c|c|c|}
\hline Catégories & Actes de langage & Icônes \\
\hline Salutation & Saluer & 3 \\
\hline \multirow[t]{3}{*}{ Initiatif } & Proposer & 2 \\
\hline & Demander 5 & $?$ \\
\hline & Affirmer & S \\
\hline \multirow[t]{2}{*}{ Réactif } & Répondre & $!$ \\
\hline & Questionner & 7 \\
\hline \multirow[t]{2}{*}{ Évaluatif } & Approuver & s \\
\hline & Désapprouver & E9 \\
\hline \multirow[t]{2}{*}{ Auto-réactif } & Préciser & $\mathbf{P}$ \\
\hline & Rectifier & $\mathbf{R}$ \\
\hline
\end{tabular}

Afin de faciliter le travail de sélection des actes de langage par l'utilisateur, nous avons cherché à définir un nombre restreint d'actes distants sémantiquement. Nous arrivons à dix actes au total. Cet ensemble d'actes a été choisi dans le but d'être facilement exploitable par les apprenants et d'être suffisant pour l'analyse que nous voulons en faire, c'est-à-dire pour la détermination des profils comportementaux. Enfin, nous rappelons que le Tableau 2 est le résultat d'une confrontation de notre outil de conversation avec les utilisateurs lors d'une première expérimentation de l'environnement SPLACH (cf. partie 5).

\subsection{Enchaînement des actes de langage dans l'outil de conversation}

Comme nous l'avons déjà exposé, les actes de langage ne sont pas indépendants les uns des autres. Ainsi, dans une conversation, les actes de langage obéissent à des

5. Des actes comme «demander» et «questionner» peuvent paraitre identiques mais ils ne seront pas disponibles en même temps car ne faisant pas partie de la même catégorie. 
contraintes d'enchaînement (cf. partie 3.3). Nous utilisons l'idée des paires adjacentes pour atteindre deux de nos objectifs: faciliter la structuration des conversations et contribuer à limiter les sélections inappropriées d'actes de langage.

Nous nous sommes beaucoup inspirés des travaux de Clark et Shaefer [ClarkSchaefer89] pour déterminer les contraintes d'enchaînements entre les actes de langage. Nous reprenons leur idée de paires adjacentes. Cependant, nous ne limitons pas la deuxième partie de la paire à un seul acte, nous proposons une liste des actes possibles. Le Tableau 3 décrit les paires adjacentes retenues.

Tableau 3. Les paires adjacentes dans l'outil de conversation proposé

\begin{tabular}{|c|c|c|c|}
\hline \multicolumn{2}{|c|}{ Types de paires adjacentes } & \multicolumn{2}{|c|}{ Exemples } \\
\hline Première partie & Deuxièmes parties & Intervention de A & Réaction de B \\
\hline Proposer & $\begin{array}{l}\text { Approuver } \\
\text { Désapprouver } \\
\text { Questionner }\end{array}$ & $\begin{array}{l}\text { - Je propose qu'on } \\
\text { écrive le document. }\end{array}$ & $\begin{array}{l}\text { - Oui, d'accord. } \\
\text { - Non, pas tout de suite. } \\
\text { - Quel document? }\end{array}$ \\
\hline Demander & $\begin{array}{l}\text { Répondre } \\
\text { Questionner }\end{array}$ & $\begin{array}{l}\text { - As-tu rempli ton } \\
\text { document? }\end{array}$ & $\begin{array}{l}\text { - Oui, je l'ai fait. } \\
\text { - Quel document? }\end{array}$ \\
\hline Affirmer & $\begin{array}{l}\text { Approuver } \\
\text { Désapprouver } \\
\text { Questionner }\end{array}$ & $\begin{array}{l}\text { - Je veux faire cette } \\
\text { tâche. }\end{array}$ & $\begin{array}{l}\text { - D'accord. } \\
\text { - Je l'avais déjà prise ! } \\
\text { - Quelle tâche? }\end{array}$ \\
\hline Répondre & $\begin{array}{l}\text { Approuver } \\
\text { Désapprouver } \\
\text { Questionner }\end{array}$ & $\begin{array}{l}\text { - Le document de } \\
\text { l'étape d'analyse. }\end{array}$ & $\begin{array}{l}\text { - Ah, oui. } \\
\text { - Tu te trompes d'étape. } \\
\text { - Où est ce document? }\end{array}$ \\
\hline Questionner & $\begin{array}{l}\text { Répondre } \\
\text { Questionner }\end{array}$ & - Quel document? & $\begin{array}{l}\text { - Celui d'analyse. } \\
\text { - Il y en a plusieurs? }\end{array}$ \\
\hline Approuver & $\begin{array}{l}\text { Approuver } \\
\text { Désapprouver } \\
\text { Questionner }\end{array}$ & $\begin{array}{l}\text { - Je suis d'accord avec } \\
\text { l'analyse de Claude. }\end{array}$ & $\begin{array}{l}\text { - Moi aussi, d'accord. } \\
\text { - Je ne suis pas d'accord. } \\
\text { - Quelle analyse? }\end{array}$ \\
\hline Désapprouver & $\begin{array}{l}\text { Approuver } \\
\text { Désapprouver } \\
\text { Questionner }\end{array}$ & $\begin{array}{l}\text { - Je ne suis pas } \\
\text { d'accord avec l'analyse } \\
\text { de Claude? }\end{array}$ & $\begin{array}{l}\text { - Oui, moi non plus. } \\
\text { - Moi, je suis d'accord. } \\
\text { - Pourquoi? }\end{array}$ \\
\hline Saluer & Saluer & - Bonjour Benoît. & - Bonjour Anne. \\
\hline
\end{tabular}

Dans ce tableau, la deuxième partie d'une paire correspond à une réaction d'un interlocuteur différent de celui ayant effectué la première partie de cette paire. De ce fait, nous n'avons pas indiqué les actes auto-réactifs qui correspondent à la réaction d'un utilisateur à une de ses propres interventions («préciser » ou « rectifier).

La Figure 1 représente un graphe de successions ou grammaire des actes de langage retenus. Ce graphe est en fait une autre vue des paires adjacentes que nous avons définies précédemment. Le nœud de gauche représente l'initiation d'une nouvelle discussion. Ainsi, un utilisateur peut commencer une discussion par une salutation («saluer») ou par un acte initiatif («demander», «proposer» ou «affirmer»). Pour prendre un autre exemple, à partir d'une proposition, un 
utilisateur peut «questionner» cette proposition ou l'évaluer («approuver» ou «désapprouver»). Pour les actes auto-réactifs, lorsqu'un utilisateur a fait une intervention, quel qu'en soit le type, il peut réagir à sa propre intervention en précisant ou rectifiant ce qu'il vient de dire.

Insérer ici figure 1 : george-fig1.jpg

Figure 1. Graphe de successions des actes de langage

Nous pouvons par ailleurs remarquer que le graphe de successions ne présente pas de nœud de fin. Cette caractéristique vise à permettre aux débats de se dérouler. Cet aspect est important dans un contexte d'apprentissage pour favoriser l'approfondissement des idées (ici l'objectif n'est pas que les utilisateurs trouvent un accord le plus rapidement possible mais qu'ils puissent échanger et partager leurs connaissances). Un fil de discussion se termine lorsqu'il n'y a plus de réaction à un message (par exemple s'il y a un accord ou une réponse satisfaisante).

\subsection{Représentation arborescente des messages}

Un grand nombre d'outils de communication écrite synchrone existe sur le marché (outils de chat). Cependant, ces outils n'ont pas beaucoup évolué depuis une vingtaine d'années et demeurent peu adaptés pour des conversations soutenues. Leur fonctionnement repose sur un empilement des messages des utilisateurs de façon temporelle. Nous formulons des critiques à ce fonctionnement. Cet empilement de messages les uns à la suite des autres selon leur ordre d'arrivée pose des problèmes dans un contexte de conversation synchrone. En effet, le temps nécessaire à la composition des textes (temps de frappe) ne permet pas de répondre de façon immédiate à un message. De ce fait, les temps de latence provoquent des imbrications des interventions qui rendent le suivi de la discussion difficile. Deux messages peuvent se retrouver proches à l'interface alors qu'ils ne sont pas forcément liés et, à l'inverse, deux messages en relation peuvent être séparés par d'autres messages. La conséquence de ce défaut majeur est d'avoir une conversation délicate à suivre et à mener. Des résultats d'analyse de discussions médiatisées par des outils de chats ont montré que celles-ci étaient très souvent incohérentes et qu'on trouvait de nombreux messages de « réparation » de la discussion [Herring99].

Afin de pallier ce problème d'imbrication, nous proposons de munir l'outil de conversation d'une représentation arborescente des messages. L'organisation des messages sous forme arborescente est beaucoup utilisée dans les forums de discussion asynchrones. Le principe est de lier chaque message à celui auquel il répond ou réagit. Les nouveaux sujets de discussion sont placés à la racine de l'arbre, les autres se raccrochant aux messages existants. L'avantage de cette représentation est de tenir compte des fils de discussion et donc des sujets de conversation. Peu de recherches ont été menées sur la représentation arborescente des conversations synchrones médiatisées. Depuis la création de notre outil, nous avons néanmoins trouvé un autre travail de recherche allant dans ce sens. Ainsi, un 
prototype d'interface nommée Threaded Chat a été développé dans les laboratoires de recherche de Microsoft ${ }^{\odot}$ [Smithandal00]. Une étude expérimentale de cet outil a permis de voir qu'il facilitait notamment l'aide à la prise de décision. Cependant, dans cet outil, chaque message ne s'affiche que sur une seule ligne ce qui oblige l'utilisateur à faire défiler le message horizontalement pour le lire dans sa totalité. L'outil que nous avons conçu (cf. partie suivante) n'a pas ce problème ergonomique grâce à un affichage des messages sur plusieurs lignes lorsque cela est nécessaire. Par ailleurs, cette représentation arborescente des messages contribue à faciliter l'utilisation des actes de langages appropriés car elle permet de les proposer de manière contextuelle.

\subsection{L'outil de conversation proposé}

À partir de tous les éléments définis ci-dessus, un outil de conversation a été conçu et développé. Il se présente sous la forme d'une arborescence d'interventions. Chaque nœud de l'arbre est le début d'un fil de discussion. La Figure 2 représente une copie d'écran de l'outil. Nous pouvons y voir un fil de discussion contenant six interventions. Chaque intervention commence par une icône (cf. Tableau 2) symbolisant l'acte de langage sélectionné par l'utilisateur. Pour intervenir, l'utilisateur clique soit sur un message existant, soit à la fin de la conversation sur la ligne « cliquer ici pour commencer une nouvelle discussion». Dans les deux cas un menu apparaît près de la souris et propose la liste des actes possibles (d'après les enchaînements spécifiés dans la partie 4.3). L'utilisateur sélectionne alors l'acte souhaité puis saisie le texte de son intervention de façon libre. Le message est alors insérée à la bonne place dans l'arborescence. Dans l'exemple ci-dessous, un clique sur une question de Michel a fait apparaitre un menu déroulant permettant de « répondre » ou de «questionner».

Insérer ici figure 2 : george-fig2.jpg

Figure 2. L'outil de conversation textuelle synchrone semi-structurée

Cet outil a été intégré à l'environnement support de projet collectif à distance SPLACH. Toutes les données issues des conversations sont donc analysables par les agents d'analyse de l'environnement SPLACH [George01]. Nous présentons dans la partie suivante la détermination automatique des profils de comportement sociaux à partir des données recueillies.

\subsection{Calcul des profils comportementaux}

À partir des spécifications précédentes concernant la conception d'un outil de conversation, il nous est possible de concevoir un système d'analyse automatique afin d'identifier les quatre profils de comportements types relevés par Pléty: animateur, vérificateur, quêteur et indépendant. Il est toujours délicat de tenter de modéliser le comportement de l'être humain. Notre objectif n'est pas ici d'obtenir un 
modèle complet de l'apprenant comme c'est le cas dans les tuteurs intelligents visant à modéliser les connaissances mais nous souhaitons disposer d'une image du comportement social lors de conversations.

Nous présentons ici les formules utilisées par un agent de l'environnement SPLACH chargé d'analyser les réunions. Ces formules heuristiques ont été déterminées à partir des indications provenant des travaux de Pléty et ont été affinées lors d'expérimentations. Une première formule permet de calculer tout d'abord le coefficient de participation d'un participant $p$ en divisant le nombre de messages envoyés par celui-ci par le nombre moyen de messages ${ }^{6}$ envoyés par les participants. Nous avons donc la formule suivante :

$$
\operatorname{coeffParticipation}(p)=\frac{\text { nbreMessages }(p)}{\text { MoyMessages }} \times 50
$$

Si le nombre de messages envoyés par un participant est égal au nombre moyen de messages, alors le coefficient de participation de ce participant est de 50 . Un coefficient de participation proche de 0 indique une faible participation alors qu'un coefficient proche de 100 indique une forte participation dans la conversation. Pour tous nos calculs, si un coefficient dépasse 100, il est alors ramené à 100. Dans le cas présent, une valeur de 100 au coefficient de participation signifie que le participant a envoyé deux fois plus de messages que la moyenne.

Quatre formules permettent de calculer des coefficients correspondant aux quatre profils : coefficient d'animation, coefficient de vérification, coefficient de quête et coefficient d'indépendance. Par exemple, le coefficient d'animation du participant $p$ est calculé par la formule suivante :

$$
\text { coeffAnimation }(p)=\frac{\text { nbMessagesInitiatifs }(p)}{n b \text { MessagesTotal }(p)} \times \frac{\text { nbTotalActes }}{n b \text { ActesInitiatifs }} \times 50
$$

Dans cette formule, nbMessagesInitiatifs $(p)$ correspond au nombre de messages de type initiatif (i.e. proposer, affirmer, demander) envoyés par le participant et nbMessagesTotal $(p)$ correspond au nombre total de messages envoyés par ce même participant. Le rapport entre ces deux termes est multiplié par le rapport entre le nombre d'actes au total (10) et le nombre d'actes de type initiatif possibles (3). Lorsque ce coefficient se rapproche de 0 , cela signifie que le participant n'est pas animateur alors qu'un coefficient proche de 100 signale un fort comportement d'animation. Les calculs des autres coefficients (vérificateur, quêteur, indépendant) sont obtenus de manière similaire en reprenant les indications fournies par Plety et en les transformant sous forme d'équations. Nous ne prétendons pas avoir déterminé

6. Cette moyenne tient compte de la présence du participant pour ne pas fausser le calcul si un participant arrive en cours de réunion. On ajoute $1 /$ nbParticipants à la moyenne à chaque message reçu. Cette moyenne n'est donc pas forcément la même pour tous les participants. 
les équations «exactes» des résultats de Pléty, mais nous proposons des formules qui reprennent ses idées et qui ont été établies de manière heuristique.

Dès lors, les profils comportementaux sont calculés en pondérant le coefficient de participation avec un des quatre coefficients décrits ci-dessus. Un coefficient de pondération ${ }^{7}$ est appliqué pour obtenir des valeurs représentatives et pertinentes. Ainsi, le profil d'animateur du participant $p$ est calculé par la formule suivante :

$$
\operatorname{profilAnimateur}(p)=\frac{\text { coeffParticipation }(p)+2 \times \operatorname{coeffAnimation}(p)}{3}
$$

Dans ce cas, une pondération de $2 / 3$ accorde plus d'importance au coefficient d'animation par rapport au coefficient de participation. Les quatre profils sont calculés au fur et à mesure d'une conversation et évoluent au cours du temps. Nous présentons dans la partie suivante un outil permettant d'observer l'évolution de ces profils.

\section{5. Évaluation de l'outil de conversation synchrone et de la détermination automatique de comportements sociaux}

En expérimentant l'environnement SPLACH, notre but est de réaliser une évaluation formative [Paquette91] [Delozanne92] pour tester les usages, les interfaces et les outils de notre environnement. Par ailleurs, les expérimentations rentrent dans notre approche de développement incrémental et itératif. Ainsi, l'usage de l'environnement nous a permis de faire évoluer et d'affiner les spécifications de celui-ci. Une description détaillée des expérimentations de l'environnement SPLACH se trouve dans [George01]. Nous nous centrons dans cette partie sur l'évaluation de l'outil de conversation textuelle synchrone.

\subsection{Contextes d'expérimentation}

L'un des objectifs de notre recherche est d'effectuer des expérimentations dans des contextes réels de formation pour ne pas nous limiter à l'observation de l'utilisation des fonctionnalités techniques mais pour observer les usages. Deux séries d'expérimentations ont été menées. La première, réalisée avec quinze élèves provenant de trois collèges, nous a permis de tester l'outil de conversation textuelle synchrone et de l'améliorer. Les améliorations ont portées sur l'interface et sur les actes de langages proposés. Nous avons ainsi ajouté les actes auto-réactifs (préciser et rectifier) ainsi qu'un acte initiatif (proposer). Nous ne nous étendons pas davantage sur cette expérimentation visant à faire évoluer un prototype pour nous concentrer sur la deuxième expérimentation.

7. Ces coefficients de pondération ont été déterminés suite à des tests et expérimentations. 
Une deuxième expérimentation de SPLACH et de l'outil de conversation synchrone a été réalisée dans un contexte de téléformation à la Télé-université du Québec. Six étudiants suivant des cours de programmation à distance ont formé deux équipes-projets pendant six semaines. L'objectif global était de réaliser un programme en Pascal de manière collective. Cette expérimentation avait plusieurs objectifs dont l'un était d'évaluer l'outil de conversation semi-structurée et la méthode de calcul des profils comportementaux.

Les données recueillies lors de cette expérimentation sont de deux natures : les traces informatiques et les réponses à des questionnaires. L'environnement SPLACH garde des traces des actions des utilisateurs dans le système. Nous avons ainsi la possibilité d'avoir des données sur les connexions, sur les activités réalisées et sur les communications effectuées. Pour ce qui nous concerne ici, le système garde des traces sur les actions des utilisateurs dans l'outil de conversation synchrone : liste des participants aux réunions synchrones, messages envoyés, actes de langage sélectionnés et fils de discussion.

En fin d'expérimentation, les étudiants ont rempli un questionnaire que nous avions élaboré pour recueillir des informations plus qualitatives. Les questions étaient donc très ouvertes. Par ailleurs, nous avons conçu un autre questionnaire pour le tuteur afin de recueillir ses impressions à l'issue de l'expérimentation.

\subsection{Résultats}

D'une manière générale, les étudiants de notre expérimentation ont apprécié l'outil de conversation synchrone et n'ont pas trouvé gênant le fait de sélectionner un acte de langage avant d'envoyer un message. Il ressort également du questionnaire que l'outil est simple d'utilisation et facile à prendre en main. Une analyse manuelle de l'utilisation des actes de langage nous montre que les étudiants les ont très souvent utilisés à bon escient (seulement 10 à $15 \%$ des actes sélectionnés ne correspondent pas au contenu du message). D'un point de vue ergonomique, la sélection des actes par menu déroulant contextuel, issue de la mise en œuvre du graphe de succession, paraît pertinente. Par ailleurs, les utilisateurs n'ont pas trouvé qu'il manquait d'actes de langage pour typer les messages. Une différence existe entre les apprenants et le tuteur : les apprenants n'ont pas trouvé d'utilité particulière dans la sélection d'actes de langage alors que le tuteur pense que ce principe est très utile au développement des idées. Selon le tuteur, l'outil facilite la discussion textuelle et l'approfondissement des idées.

Contrairement à la remarque de Cerratto [Cerratto99] qui signalait qu'à distance les échanges étaient souvent minimaux (initiatif-réactif), nous avons constaté beaucoup d'échanges complets (initiatif-réactif-évaluatif) dans les conversations. Cet aspect évaluatif signale un engagement plus important des locuteurs [KerbratOrecchioni92]. Nous interprétons ce résultat par le fait que l'outil incite à évaluer ce qui se dit (approuver, désapprouver) et également par l'activité de projet qui pousse à prendre position. 
L'expérimentation a permis de montrer que les profils comportementaux calculés étaient fidèles à ce qui se passait dans les conversations. Les formules de calculs élaborés semblent donc pertinentes par rapport à notre contexte d'usage. La Figure 3 montre un outil qui permet de visualiser l'évolution des profils comportementaux au cours d'une conversation. Cet outil a pour l'instant été conçu dans un objectif d'observation expérimentale mais il pourrait être fourni au tuteur pour l'assister dans le suivi des apprenants ou aux apprenants eux-mêmes pour leur donner une vue sur leurs propres comportements.

Insérer ici la figure 3 : george-fig3.jpg

Figure 3. L'outil de visualisation des profils comportementaux

Chaque graphe correspond à un type de profil particulier et les courbes représentent l'évolution dans le temps des profils des utilisateurs présents à la réunion. Nous avons donc quatre graphes : animateur, vérificateur, quêteur et indépendant. Sur chaque graphe, nous avons une courbe pour chacun des participants à la conversation, pour trois participants dans notre exemple. Les profils calculés sur 100 sont représentés en ordonnée et le temps est en abscisse. Pour information, la durée totale de la conversation prise en exemple est d'environ une heure et il y a eu 66 messages échangés. L'outil de visualisation permet de faire des interprétations. Par exemple, le graphe «Animateur » de la Figure 3 signale que l'utilisateur Luc a majoritairement tenu un rôle d'animateur lors de cette réunion alors que Michel a été le «moins animateur». Par ailleurs, nous pouvons voir que Michel a été le plus « quêteur » et qu'aucun participant n'a vraiment été « indépendant ».

Les résultats de ces analyses automatiques de réunions n'étaient pas à disposition du tuteur durant cette expérimentation. Cependant, ayant vu a posteriori ces résultats, le tuteur qui encadrait les étudiants a déclaré que cet outil lui aurait été utile pour avoir une vue sur les réunions auxquelles il n'avait pas assisté.

\section{Conclusion et perspectives}

Nous avons présenté notre travail visant une double objectif : faciliter les activités collectives entre apprenants et permettre leur suivi. Nous nous sommes centrer sur un cas particulier : les activités de discussions synchrones sous forme textuelle. Nous avons pour cela conçu un outil de conversation synchrone particulier facilitant, d'une part, le déroulement des conversations et, d'autre part, rendant possible l'analyse automatique de celles-ci dans le but de déterminer des profils comportementaux sociaux.

Les résultats obtenus lors de l'expérimentation à la Télé-université, semblant confirmer notre hypothèse de retrouver les profils comportementaux relevés par Pléty dans les groupes d'apprenants travaillant à distance, nous encouragent à 
poursuivre dans cette voie. Il est vrai que notre système pourrait être affiné en prenant d'autres variables en compte. Par exemple, une intervention d'un animateur se caractérise aussi par le fait qu'elle entraîne souvent des réactions. Cependant, notre volonté était d'avoir des calculs peu complexes pour le moment et ne mettant pas en jeu un trop grand nombre de paramètres.

Par ailleurs, la richesse des analyses de Pléty vient du fait qu'il ne se limite pas seulement au plan verbal mais qu'il prend aussi en compte la gestualité dans l'interaction. À ce sujet, il distingue les gestes et les regards communicatifs (adressés à un destinataire) des gestes non-communicatifs (postures, gestes d'auto-contacts). Un travail avec des chercheurs en traitement d'image est envisageable pour la prise en compte de la gestualité dans la détermination des profils de comportements à distance. Cependant, les gestes relevés par Pléty dans ses analyses de groupe en face-à-face sont difficilement transposables tels quels à distance (gestes et regards vers les autres). En revanche, les gestes d'auto-contact et les postures peuvent demeurer révélateurs de comportements.

Nous tenons à signaler le biais que peut entraîné l'outil informatique de conversation dans le calcul des profils comportementaux. En effet, comme tout outil, celui-ci modifie l'activité de l'utilisateur. De ce fait, nous analysons ici une conversation médiatisée par un outil. Le calcul que nous faisons est donc effectué par rapport à l'activité de conversation dans un contexte précis et ne prétend en aucun cas refléter les comportements des utilisateurs d'une façon générale.

Une perspective à nos travaux concerne l'approfondissement de l'étude sur l'outil de visualisation de profils de comportementaux et d'une façon plus large sur l'instrumentation du formateur dans son rôle d'encadrement des apprenants dans un contexte d'apprentissage collectif. En effet, les expérimentations nous ont enseigné que le tuteur doit souvent entrer en contact par forum ou courrier électronique avec les apprenants afin de comprendre le déroulement des activités. Il serait utile de lui fournir des outils appropriés à son activité de suivi pour pallier cette surcharge de travail. Nous nous orientons vers la conception d'outils donnant au tuteur une vue synthétique et organisée des activités individuelles et collectives des apprenants ainsi que de leurs communications.

Le travail présenté dans cet article a servi de base à un projet mené actuellement par le laboratoire d'informatique de l'Université du Maine. Une équipe de chercheur travaille sur un outil de discussion qui reprend le principe de structuration sous la forme d'arborescence d'énoncés et d'enchaînement d'actes de langage. L'outil, nommé Oscar, est un outil de recherche dont les actes de langage et les grammaires d'enchaînement sont paramétrables [Delium03]. De plus, Oscar permet à la fois les discussions synchrones et asynchrones. Nous pensons néanmoins que les actes de langage et les enchaînements ne peuvent être les mêmes en synchrone et en asynchrone car les natures de ces deux modes de discussion sont bien différentes. Dans le cas du synchrone, les messages sont courts et l'acte de langage directeur est facilement identifiable par l'utilisateur. Dans le cas de conversations asynchrones, les énoncés sont bien souvent beaucoup plus longs et comprennent en fait plusieurs 
messages, donc plusieurs actes de langage directeurs. Il est alors beaucoup plus difficile de demander à l'utilisateur de typer son intervention. L'utilisation d'Oscar devrait permettre de tester ce point.

\section{Remerciements}

L'auteur a réalisé les travaux de recherche présentés dans cet article dans le cadre de son travail de thèse au Laboratoire d'Informatique de l'Université du Maine. Cette recherche a été en partie réalisée dans le cadre du collectif scientifique LÉA qui a été soutenu par la commission permanente de coopération franco-québecoise. L'auteur tient à remercier toutes les personnes qui ont permis de réaliser les expérimentations en situation réelle.

\section{Bibliographie}

[Austin62] Austin, J. L. (1962). How to do Things with Words. Oxford University Press.

[BakerLund97] Baker, M. J., Lund, K. (1997). Promoting reflective interactions in a computer-supported collaborative learning environment. Journal of Computer Assisted Learning, 13, 175-193.

[Bilange91] Bilange, É. (1991). Modélisation du dialogue oral finalisé personne-machine par une approche structurelle. Thèse de Doctorat en Informatique, Université de Rennes I.

[Cerratto99] Cerratto, T. (1999). Activités collaboratives sur réseau, une approche instrumentale de l'écriture en collaboration. Thèse de Doctorat en Psychologie cognitive, Université Paris 8.

[ClarkSchaefer89] Clark, H. H., Schaefer, E. F. (1989). Contributing to Discourse. Journal of Cognitive Science, 13, 259-294.

[ColineauMoulin99] Colineau, N., Moulin, B. (1999). Détermination d'actes de dialogue suivant une approche connexionniste. In Analyse et Simulation de Conversations, Moulin, B., Delisle, S., Chaib-draa, B., (Eds.), Lyon, L'interdisciplinaire, 243-271.

[Delium03] Delium, C. (2003). OSCAR, un environnement de communication médiatisée structurée par les actes de langages. Actes de la conférence EIAH 2003, Strasbourg, (1516 avril), $127-138$.

[Delozanne92] Delozanne, E. (1992). Explication en EIAO : études à partir d'Elise, un logiciel pour s'entraîner à une méthode de calcul de primitives. Thèse de Doctorat en Informatique, Université du Maine.

[Dewey22] Dewey, J. (1922). L'école et l'enfant. Paris, Delachaux et Niestlé.

[Dillenbourganda196] Dillenbourg, P., Baker, M. J., Blaye, A., C.O'Malley (1996). The evolution of research on collaborative learning. Learning in Humans and Machines: Towards an Interdisciplinary Learning Science, Spada, H., Reimann, P. (Eds.), 189- 211.

[DoiseMugny81] Doise, W., Mugny, G. (1981). Le développement social de l'intelligence. Paris, InterEditions. 
[Floresandal88] Flores, F., Graves, M., Hartfield, B., Winograd, T. (1988). Computer Systems and the Design of Organizational Interaction. ACM Transactions on Office Information Systems, 6 (2), 153-172.

[Freinet77] Freinet, C. (1977). Les Techniques Freinet de l'école moderne. Bourrelier (Ed.) librairie Armand Colin, Carnet de pédagogie pratique.

[George01] George, S. (2001). Apprentissage collectif à distance. SPLACH : un environnement informatique support d'une pédagogie de projet. Thèse de Doctorat en Informatique, Université du Maine.

[George02] George, S. (2002). SPLACH: a Computer Environment Supporting Distance Project-Based Learning. Proceedings of the World Conference on Educational Multimedia, Hypermedia \& Telecommunications ED-MEDIA 2002, Denver, Colorado, USA, (June 24-29), 588-593.

[GeorgeLeroux01] George, S., Leroux, P. (2001). Un environnement support de projets collectifs entre apprenants : SPLACH. Actes des sixièmes journées francophones Environnements Interactifs d'Apprentissage avec Ordinateur (EIAO'2001), Paris, (26-27 avril), Hermès, 49-60.

[GeorgeLeroux02] George, S., Leroux, P. (2002). Un environnement pédagogique dédié aux projets collectifs à distance intégrant une assistance aux apprenants et au chef de projet. Actes de la 3ème conférence internationale sur les Technologies de l'Information et de la Communication dans l'Enseignement d'Ingénieurs et dans l'industrie (TICE 2002), Lyon, France, (13-15 octobre), 289-295.

[Herring99] Herring, S. (1999). Interactional Coherence in CMC. Journal of ComputerMediated Communication, McLaughlin, M. L., Rafaeli, S. (Eds.), 4 (4), http://www.ascusc.org/jcmc/vol4/issue4/.

[JermannSchneider97] Jermann, P., Schneider, D. (1997). Semi-structured interface in collaborative problem-solving. First Swiss Workshop on distributed and parallel systems.

[Jermannandal01] Jermann, P., Soller, A., Muehlenbrock, M. (2001). From mirroring to guiding: A review of state of the art technology for supporting collaborative learning. Proceedings of the European Conference on Computer-Supported Collaborative Learning (Euro-CSCL 2001), Maastricht, Netherlands, (march 22-25), 324-331.

[KendonFerber73] Kendon, A., Ferber, A. (1973). A description of some human greetings. in Comparative ecology and behaviour of primates, Michael, R. P., Crook, J. H., (Eds.), London, Academic Press, 591-668.

[Kerbrat-Orecchioni92] Kerbrat-Orecchioni, C. (1992). Les interactions verbales. Paris, Armand Colin, vol. 2.

[Labat02] Labat, J.-M. (2002). EIAH : quel retour d'informations pour le tuteur ? Actes de la 3ème conférence internationale sur les Technologies de l'Information et de la Communication dans l'Enseignement d'Ingénieurs et dans l'industrie (TICE 2002), Lyon, France, (13-15 novembre), 81-88.

[Laver81] Laver, J. (1981). Linguistic routines and politeness: Greeting and parting. in Conversational routines, Coulmas, F., (Ed.), New York, Mounton, 289-305. 
[Linard01] Linard, M. (2001). Concevoir des environnements pour apprendre : l'activité humaine, cadre organisateur de l'interactivité technique. Revue Sciences et Techniques Educatives, 8 (3-4), 211-238.

[Luzzati95] Luzzati, D. (1995). Le dialogue verbal homme-machine. Paris, Masson.

[McManusAiken95] McManus, M. M., Aiken, R. M. (1995). Using an Intelligent Tutor to Facilitate Collaborative Learning. Innovating Adult Learning with Innovative Technologies, Davies, G., Collis, B. (Eds.), Elsevier Science, 49-64.

[Moeschler94] Moeschler, J. (1994). Analyse du discours et analyse conversationnelle. In Dictionnaire Encyclopédique de pragmatique, Moeschler, J., Reboul, A., (Eds.), Edition du Seuil, 471-492.

[Paquette91] Paquette, G. (1991). Métaconnaissance dans les environnements d'apprentissage. Thèse de Doctorat en Informatique, Université du Maine.

[Pléty96] Pléty, R. (1996). L'apprentissage coopérant. Lyon, ARCI Presse Universitaire, Collection Ethologie et psychologie des communications.

[Pléty98] Pléty, R. (1998). Comment apprendre et se former en groupe. Paris, Retz.

[Rheingold93] Rheingold, H. (1993). Les communautés virtuelles. Paris, Addison-Wesley France.

[Robertsonanda198] Robertson, J., Good, J., Pain, H. (1998). BetterBlether: The Design and Evaluation of a Discussion Tool for Education. International Journal of Artificial Intelligence in Education, 9, 219-236.

[Roulet86] Roulet, E. (1986). Complétude interactive et mouvements discursifs. Cahier de Linguistique Française, 7, 189-206.

[Rouletanda185] Roulet, E., Auchlin, A., Moeschler, J., Schelling, M., Rubattel, C. (1985). L'articulation $d u$ discours en français contemporain. Berne, Peter Lang, Collection Sciences pour la communication.

[Scapin86] Scapin, D. (1986). Guide ergonomique de conception des interfaces. Rapport de recherche $\mathrm{n}^{\circ} 77$, Rocquencourt, INRIA.

[Searle72] Searle, J. R. (1972). Les actes de langage. Hermann, Editeurs des sciences et des arts, Collection Savoir : Lettres.

[Self88] Self, J. A. (1988). Bypassing the Intractable Problem of Student Modelling. Proceedings of the 1st International Conference on Intelligent Tutoring System, Montréal, Canada, (june 1-3), 18-24.

[Smithanda100] Smith, M., Cadiz, J. J., Burkhalter, B. (2000). Conversation Trees and Threaded Chats. Proceedings of the ACM Conference on Computer Supported Cooperative Work (CSCW'00), Philadelphia, Pennsylvania, USA, (december 2-6), 97105.

[Solleranda199] Soller, A., Linton, F., Goodman, B., Lesgold, A. (1999). Toward Intelligent Analysis and Support of Collaborative Learning Interaction. Proceedings of the ninth International Conference on Artificial Intelligence in Education, Le Mans, (July 18-23), IOS Press, 75-82. 
[Suchman94] Suchman, L. A. (1994). Do Categories Have Politics? - The language/action perspective reconsidered. In Computer-Supported Cooperative Work, Kluwer Academic Publishers, vol. 2, 177-190.

[Vygotski34] Vygotski, L. S. (1934). Pensée et langage. Cambridge, MA, MIT press.

[Winograd87] Winograd, T. (1987). A Language/Action Perspective on the Design of Cooperative Work. Human-Computer Interaction, 3 (1), 3-30.

Sébastien George est maître de conférences à l'INSA de Lyon depuis 2002 et rattaché au laboratoire ICTT (Interaction Collaborative, Téléformation, Téléactivités). Après l'obtention d'un doctorat en informatique à l'Université du Maine, il a travaillé au centre de recherche LICEF de la Télé-université du Québec dans le cadre d'un post-doctorat. Ses travaux portent sur les environnements informatiques support à l'apprentissage collectif. 